Schmerz 2016 $30: 1-2$

DOI 10.1007/s00482-015-0086-1

Online publiziert: 25. Januar 2016

(c) Deutsche Schmerzgesellschaft e.V. Published by Springer-Verlag Berlin Heidelberg - all rights reserved 2016

\section{CrossMark}

\author{
L. Radbruch ${ }^{1,2} \cdot$ M. Schäfer ${ }^{3,4}$ \\ ${ }^{1}$ Klinik für Palliativmedizin, Universitätsklinikum Bonn, Bonn, Deutschland \\ 2 Deutsche Gesellschaft für Palliativmedizin e. V., Berlin, Deutschland \\ ${ }^{3}$ Klinik für Anästhesiologie und operative Intensivmedizin, Charité Universitätsmedizin \\ Berlin, Campus Virchow-Klinikum, Berlin, Deutschland \\ ${ }^{4}$ Deutsche Schmerzgesellschaft e. V., Berlin, Deutschland
}

\title{
Cannabis als Medikament
}

\section{Ein Schwerpunktthema}

ist, wenn andere verfügbare Therapien nicht angemessen wirken, übernehmen die Krankenkassen die Kosten für Cannabisprodukte - bei einer Dosis von $10 \mathrm{mg}$ THC pro Tag sind es zwischen 230 und $720 €$ pro Monat - bei anderen Erkrankungen in der Regel nicht.

Patienten können beim BfArM auf Antrag eine betäubungsmittelrechtliche Ausnahmeerlaubnis zum Erwerb von Medizinalhanf oder Cannabisextrakt zur Anwendung in einer ärztlich begleiteten Selbsttherapie erhalten. Medizinalhanf kann von einer Apotheke über den pharmazeutischen Großhandel aus den Niederlanden bezogen und von denjenigen, die eine Ausnahmeerlaubnis haben, in der Apotheke erworben werden. Mittlerweile wurde in mehr als 500 Fällen eine solche Ausnahmegenehmigung erteilt. Allerdings liegen die Kosten für die Therapie mit importiertem Medizinalhanf oft noch höher und können bis zu $1500 €$ im Monat betragen.

\section{Eigenanbau oder Apothekenausgabe?}

Zudem besteht die Möglichkeit einer Ausnahmeerlaubnis durch das Bundesinstitut für Arzneimittel und Medizinprodukte (BfArM) zur Verwendung von Cannabisblüten aus der Apotheke. $\mathrm{Zu}$ gelassen und von den Krankenkassen erstattet wird in Deutschland bisher nur eine Mischung von THC und CBD als Spray zur Behandlung der Spastik bei multipler Sklerose.

Obwohl grundsätzlich eine Kostenübernahme durch die Krankenkassen auch bei anderen Indikationen möglich
Wenn Patienten diese hohen Therapiekosten nicht aufbringen konnten, wurde immer wieder eine Genehmigung zum Anbau von Cannabis zum Eigenbedarf gefordert. Im Juli 2014 hat das Kölner Verwaltungsgericht einen solchen Eigenanbau für drei schwerkranke Patienten erlaubt. Nach der Begründung des Gerichts ist ein solcher Eigenanbau dann berechtigt, wenn der Patient austherapiert ist, es keine Behandlungsalternative zu Cannabis gibt und Apotheken- cannabis unerschwinglich ist. Das Urteil ist allerdings nicht rechtskräftig, da die Bundesregierung Berufung eingelegt hat.

In dieser rechtlichen Auseinandersetzung verwundert es uns, dass die Verweigerung der Kostenübernahme für Rezeptur- oder Fertigarzneimittel durch die Krankenkassen als Begründung dient, den Einsatz von Medizinalhanf in Einzelfällen zu genehmigen. Es gibt keine Studien, die Medizinalhanf mit synthetischen oder teilsynthetischen Cannabinoiden vergleichen, und es gibt keine Hinweise, dass Medizinalhanf eine andere oder bessere Wirkung entfaltet als THC für die Indikationen in der Schmerzund Palliativmedizin. Warum sollte man also nicht THC als Rezeptur- oder Fertigarzneimittel für eine dosisstandardisierte Therapie einsetzen?

Im Gegenzug gibt es eine Reihe von Gründen, die Verwendung von Medizinalhanf kritisch $\mathrm{zu}$ sehen. So werden Cannabisprodukte (Marihuana, Haschisch) von Konsumenten häufig mit Tabak gemischt, mit allen bekannten Nachteilen des Tabakrauchens. Zudem ist der Gehalt an THC in getrocknetem Hanf (Marihuana), in gepresstem Harz (Haschisch) und in Haschischöl nicht standardisiert. Das Ausmaß der psychotropen Effekte nach Konsum ist daher kaum absehbar. In Deutschland gibt es bisher keinen gewerbemäßig legalen Anbau von Cannabis für medizinische Zwecke. In den Niederlanden erhielten ursprünglich zwei Bauern eine Zulassung für legalen Anbau. Mittlerweile konnte einer der beiden die strengen Auflagen und Qualitätskontrollen nicht mehr er- 
füllen, sodass die Lizenz für ihn zurückgezogen wurde und es jetzt nur noch einen Anbauer gibt (persönliche Mitteilung von Willem Scholten, früher zuständig für die Regulierung der Cannabisfreigabe für medizinische Zwecke in den Niederlanden).

Beim Eigenanbau von Cannabis durch Patienten mit chronischen Schmerzen entstehen zusätzliche Gefahren durch die möglichen starken Schwankungen des Gehalts an THC und CBD oder durch die Kontaminationsgefahr des selbst angebauten Cannabis. Ohne ausreichende Kontrollen sind in Medizinalhanf oft Verunreinigungen bis hin zur Pilzkontamination $\mathrm{zu}$ finden (persönliche Mitteilung von W. Scholten). In den vorliegenden Studien wurde Medizinalhanf verwendet, der einen standardisierten THC-Gehalt hatte und von einer nationalen Behörde zur Verfügung gestellt wurde. Der Eigenanbau von Cannabis zur Schmerztherapie durch Patienten ist deshalb nicht zu empfehlen. Die zu hohen Therapiekosten dürfen hier nicht als Begründung reichen, vielmehr müsste für Patienten, bei denen in einem Therapieversuch eine angemessene Effektivität der Therapie mit Cannabinoiden oder Medizinalhanf nachgewiesen worden ist, eine Kostenübernahme durch die Krankenkassen gewährleistet werden.

\section{Sind wir auf dem richtigen Weg?}

Die Bundesregierung hat nun ein Gesetzesvorhaben angekündigt, mit dem die Hürden für die Nutzung von Cannabis als Medikament abgesenkt werden sollen. So sollen etwa die Kosten der Behandlung von den Krankenkassen übernommen werden. Dabei ist auch die Einrichtung einer staatlichen Stelle im Gespräch, die den Anbau und Handel von Medizinalhanf überwachen soll („Cannabisagentur").

Gleichzeitig wird vom Bundesgesundheitsministerium ein Forschungsvorhaben gefördert, das den aktuellen Forschungsstand zum nichtmedizinischen Konsum und medizinischen Gebrauch von Cannabis zusammenfassen soll. Damit soll auch geklärt werden, bei welchen medizinischen Indikationen Cannabis- produkte in Zukunft in Deutschland verwendet werden können.

Die in diesem Schwerpunktheft in einer Serie von Übersichtsarbeiten zusammengefasste Studienlage zu den verschiedenen medizinischen Indikationen von Cannabinoiden ist ernüchternd und unterstützt nicht die Euphorie für einen breiten medizinischen Einsatz [1-5]. Die Erfahrungsberichte aus Israel und Kanada [6] spiegeln positive Erfahrungen wider, es werden aber auch zahlreiche Kontraindikationen für eine Cannabistherapie beschrieben. Berichtet wird auch über die zunehmende Besorgnis, dass die Unterscheidung zwischen medizinischem Einsatz und Freizeitgebrauch verwischt wird.

> Die aktuelle Datenlage kann einen Einsatz von Cannabisprodukten bei sorgfältig ausgewählten Patienten mit spezifischen Indikationen, die nicht ausreichend auf etablierte medikamentöse Therapieverfahren ansprechen, begründen.

Ein solches Therapieverfahren sollte idealerweise immer im Kontext einer multimodalen Schmerztherapie und nicht als isoliertes Therapieverfahren Anwendung finden. Bei diesen Patienten sollten die Kosten eines eng begleiteten individuellen Therapieversuchs von den Krankenkassen erstattet werden. Idealerweise sollte ein solches Vorgehen aufgrund der eingeschränkten Datenlage zur Sicherheit und Effektivität der Langzeittherapie in einem bundesweiten Register zur Qualitätssicherung begleitet werden.

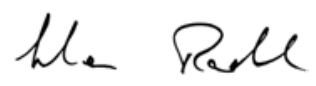

L. Radbruch

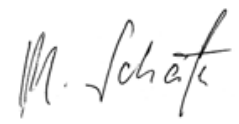

M. Schäfer

\section{Korrespondenzadresse}

Prof. Dr. L. Radbruch

Klinik für Palliativmedizin,

Universitätsklinikum Bonn

Sigmund-Freud-Str. 25, 53127 Bonn

lukas.radbruch@malteser.org
Interessenkonflikt. L. Radbruch und M. Schäfer geben an, dass kein Interessenkonflikt besteht.

\section{Literatur}

1. Fitzcharles MA, Baerwald C, Ablin J, Häuser W (2016) Efficacy, tolerability and safety of cannabinoids in chronic musculoskeletal pain (fibromyalgia syndrome, spinal pain, osteoarthritis, rheumatoid arthritis): a systematic review of randomized controlled trials. Schmerz. doi:10.1007/s00482-015-0084-3

2. Mücke $M$, Carter $D$, Cuhls $H$, Prüß $M$, Radbruch $L$, Häuser W (2016) Cannabinoide in der palliativen Versorgung. Systematische Übersicht und Metaanalyse der Wirksamkeit, Verträglichkeit und Sicherheit. Schmerz. doi:10.1007/s00482-0150085-2

3. Petzke F, Häuser W, Krumova EK (2016) Efficacy, tolerability and safety of cannabinoids for chronic neuropathic pain - a systematic review of systematic reviews. Schmerz. doi:10.1007/s00482015-0089-y

4. Tafelski S, Häuser W, Schäfer M (2016) Efficacy, tolerability and safety of cannabinoids for chemotherapy-induced nausea and vomiting - a systematic review of systematic reviews. Schmerz. doi:10.1007/s00482-015-0092-3

5. Volz S, Häuser W, Sigmund B (2016) Wirksamkeit, Verträglichkeit und Sicherheit von Cannabinoiden in der Gastroenterologie - chronisch-entzündliche Darmerkrankungen, Reizdarmsyndrom und chronische Pankreatitis: Eine systematische Übersichtsarbeit. Schmerz. doi:10.1007/s00482-0150087-0

6. Ablin J, Ste-Marie P, Schäfer M, Häuser W, Fitzcharles MA Medical use of cannabis productslessons to be learned from Israel and Canada. Schmerz. doi:10.1007/s00482-015-0083-4 